\title{
Idiopathic pericardial effusion in 2 year old labrador managed with ultrasound-guided pericardiocentesis: Case report
}

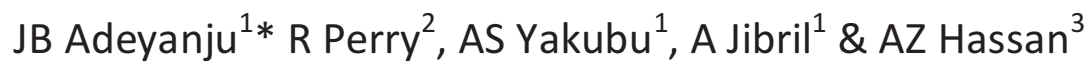 \\ ${ }^{1}$ Faculty of Veterinary Medicine, Usman Danfodiyo University, Sokoto. \\ ${ }^{2}$ College of Veterinary Medicine, Michigan State University East Lansing, Mi, 48824-1314. \\ ${ }^{3}$ Faculty of Veterinary Medicine, Ahmadu Bello University, Zaria.
}

\begin{abstract}
A two-year old, 38kg-wt male Labrador was presented for management because of progressive exercise intolerance. At presentation, rectal temperature was $38.5^{\circ} \mathrm{C}$, pulse rate was 135 beats per minute (but weak). There was ascites along with oedema of the extremities. The heart sound was muffled and pulsus paradoxus was very mild. The patient was well hydrated. Thoracic radiography revealed a globoid shaped heart occupy ing most of the equatorial thoracic volume; there was loss of details of cardiac silhouette and there was dorsal deviation of trachea at carina. Ultrasonography revealed a distinct epicardium, pericardium and a very wide anechoic space in between. Blood picture was within normal findings. Idiopathic chronic pericardial effusion was diagnosed. Ultrasound-guided pericardiocentesis was carried out using a 16 gauge over the needle catheter attached to a 3way stopcock and a $20 \mathrm{mls}$ syringe; about $65 \mathrm{mls}$ of clear effusate was aspirated. Laboratory analysis of the effusate revealed that it was a transudate. The patient was placed on $3 \mathrm{mg} / \mathrm{kg}$ furosemide, twice daily for 5 days and the patient returned to gradual exercise during hospitalization. Oedema of the extremities and ascities decreased, the appetite improved and the dog became more active. Thoracic radiography fourth week post pericardiocentesis revealed a normal cardiac silhouette. The cause of pericardial effusion was not known.
\end{abstract}

Keywords: Idiopathic, pericardial-effusion, pericardiocentesis, transudate, ultrasound-guided.

\section{Introduction}

Pericardial effusion can be life threatening in acute cases and debilitating in chronic cases. Acute effusion results from direct or indirect trauma to myocardium, coronary vessels or mitral valve; the effusate is haemorrhagic and can lead to cardic tamponade. Other known causes include cardiac neoplasia and intoxication with anticoagulant, (Petrus \& Henik, 1999; Prosek et al., 2003. An exudative pericarditis resulting from pericarditis is not common, (Carpenter et al., 2001; Heinritz et al., 2005). A transudate occurs secondary to congestive heart failure, hypoalbuminemia or idiopathic, (Tobias \& McNiel, 2008).

Acute haemorhagic pericardial effusion usually results in cardiogenic shock and death, but patients with chronic effusion have a history of progressive exercise intolerance, lethargy, hyperpnea and weakness, (Tobias, 2005).

Typical physical examination findings in patients with chronic pericardial effusion include muffled heart sound, weak femoral pulse, hyperpnea, oedema of extremities and ascites. When there is protracted loss of appetite, weight loss and dehydration are consistent findings. When distention of jugular vein is evident, pulsus padadoxus is a finding, (Tobias \& McNiel, 2008).

Diagnosis involves a combination of history, clinical examination findings, electrocardiography, radiography and echocardiography, (Sisson \& Thomas, 1999; Tobias \& McNiel, 2008). Aetiologic diagnosis involves interpretation of haematologic findings to rule out infection, cytology and analysis of effusate to differentiate transudate, exudates and haemorrhage, suggestive of neoplastic origin, (Carpenter et al., 2001; Tobias \& McNiel, 2008).

Emergency management of acute pericardial effusion and stabilization of the patient in chronic effusion is by pericardiocentesis, (Tobias \& McNiel, 2008). Pericardiectomoy had been used as curative for idiopathic pericardial effusion and palliative for effusion of neoplastic origin along with chemotherapy (Heinritz et al., 2005; Tobia \& McNiel 2008). Pericardial window and percutaneou ballon pericardiotomy had been used as palliative for effusion resulting in cardiac tamponade, (Walsh et al., 1999; Tobias, 2005) Tobias \& McNiel, 2008). 
The management and outcome of a case of idiopathic pericardial effusion in a two year old male Labrador is presented.

\section{Case Report}

A two year old $38.0 \mathrm{~kg}$-wt male Labrador was referred for management because of progressive exercise intolerance accompanied with ascites. The referring clinician had placed the patient on furosemide without remarkable improvement. The appetite was said to be marginal.

At presentation, the patient was in a good body condition but appeared listless and preferred to lie on examination table during examination. There was ascities and oedema of extremities. Capillary refill time was less than 2 seconds and the visible mucosae were pink. On auscultation, the heart beat was 135 beats pert minute, and there was hyperpnoea. There were no rales. The rectal temperature was $38.5^{\circ} \mathrm{C}$.

Blood sample was taken for complete blood count and serology. Lateral and ventro-dorsal (VD) radiographs of the thorax were taken. The patient was placed in a well ventilated cage while evaluating the laboratory results. The blood picture and serum values were within normal findings. Thoracic radiographs revealed a globoid (football) shaped

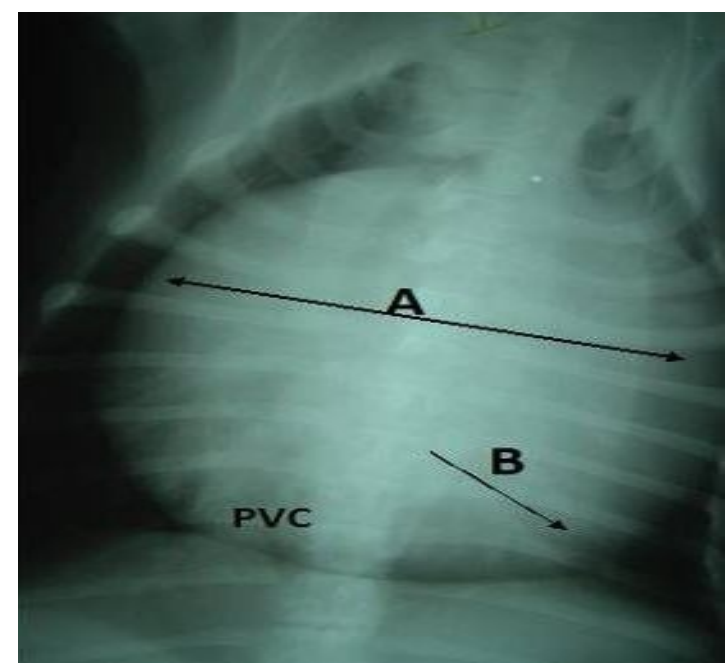

Plate I: Pre-surgical ventro-dorsal radiograph of patient. A: Disproportionate cardio-thoracic ratio Uniformly rounded cardiac silhouette B: PVC (Posterior venecava) heart, occupying most of the thoracic volume. There was loss of details of cardiac silhouette, and the trachea was elevated (lateral view); the posterior vena-cava was prominent, (Plates 1 and 2). A tentative diagnosis of pericardial effusion was made. The patient was restrained on left lateral recumbency, and the area between the third to the seventh intercostals was prepared for ultrasonography as described by Schelling et al. (1993). A lateral view ultrasonography of the heart was carried out using a linear transducer at $5 \mathrm{MHz}$. The movement of myocardium was appreciated; there was an anechoic space between the epicardium (less echogenic) and pericardium (pronounced) echogenic layer.

The right lateral thoracic wall was prepared for sterile procedure; the fifth intercostal space at the level of costochondral junction was infiltrated with $2 \%$ xylocain. A 16 guage over the needle catheter was guided by ultrasound and advanced perpendicularly to the pericardial sac (Plate 3). The needle was removed as soon as pericardial effusate exit at the hub of the needle; the catheter was slowly advanced towards epicardium to lie mid-way pericardial sac. The catheter was then attached to an extension set with a 3-way stopcock and a $20 \mathrm{cc}$ plastic syringe. About $65 \mathrm{mls}$ of clear brownish

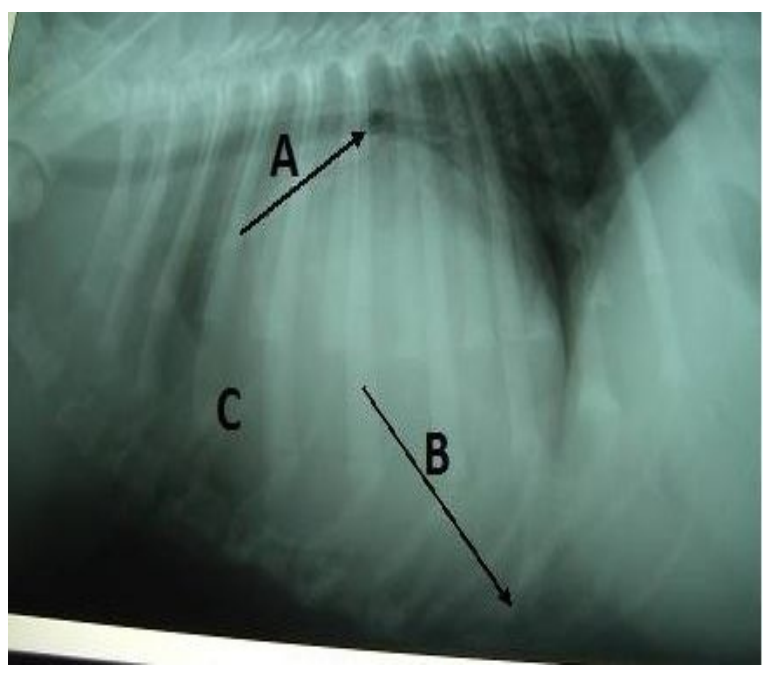

Plate II: Pre-surgical lateral radiograph of patient. A: Dorsally displaced carina.

B: loss of cardio-phrenic angle

C: Compression of apical lung lobes 


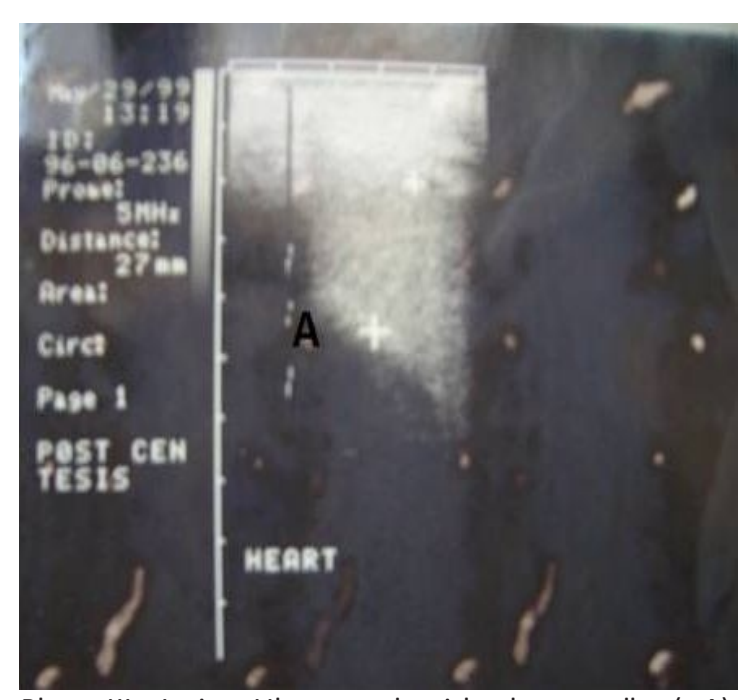

Plate III: Insitu Ultrasound with the needle ( A) advancing towards the pericardial sac

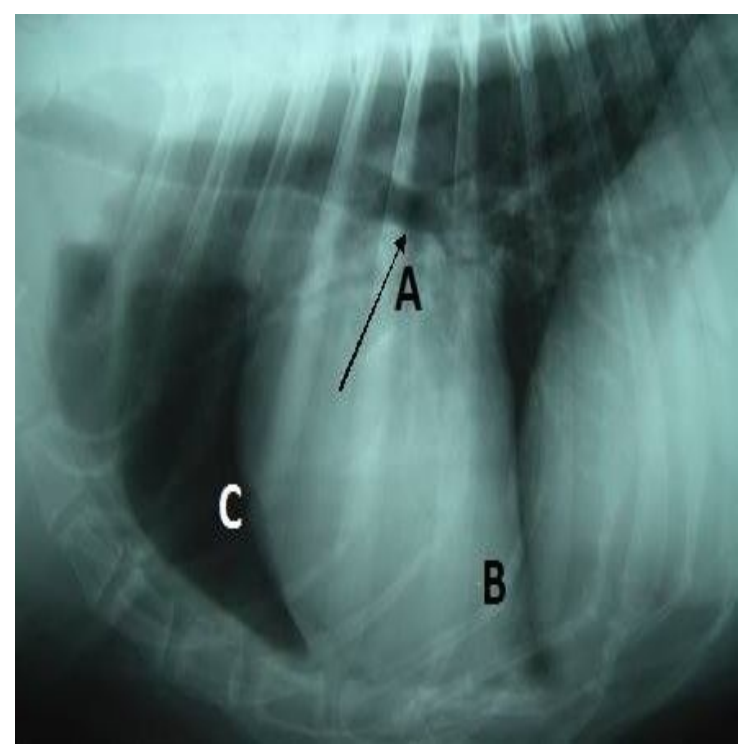

Plate V: Post-surgical lateral radiograph of patient.

A: Improved location of the carina

B: improved cardio-phrenic angle

C: Prominent apical lung lobe

effusate was aspirated which did not clot. Part of the effusate was sent to the laboratory for evaluation.

Towards the ends of aspiration, the patient started struggling and the catheter was removed. The patient was placed on $3 \mathrm{mg} / \mathrm{kg}$ furosemide, bid for 5 days during hospitalization and returned to mild walking exercise. With improved appetite and exercise tolenrance, the patient was discharged on $7^{\text {th }}$ day of hospitalization.

Thoracic radiographs taken 14 days post presentation revealed near normal cardiac

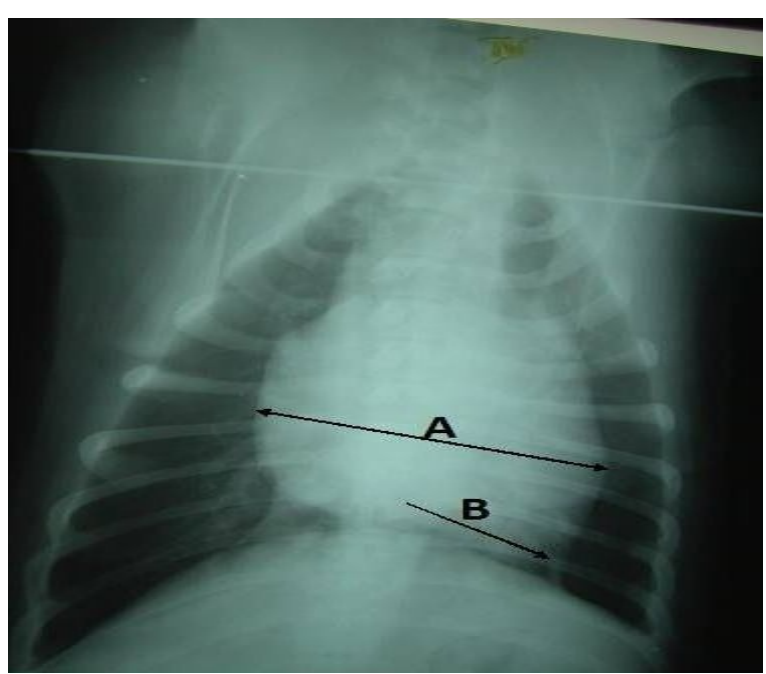

Plate IV: Post-surgical ventro-dorsal radiograph of patient.

A: Improve cardiothoracic ratio

B: Normal apex

silhouette (Plates 3 \& 4); the oedema of extremities and ascites were no longer present and the patient was active. The analysis of effusate was negative for any cell and no bacterial organism was cultured. There was no re-occurrence of the initial presenting signs six months post pericardiocentesis. Based on the response to the palliative management, absence of any obvious suspicious aetology, a diagnosis of idiopathic pericardial effusion was made. 


\section{Discussion}

Most cases of acute pericardial effusion could result in cardiac tampanade, (Prosek et al., 2003); apart from the cardio-pulmonary embarrassment, typical clinical, radiographic, electrocardiographic and ultrasonographic findings, results are difficult to be documented because of the resultant death, (Petrus \& Henik, 1999; Carpenter et al, 2001; Prosek et al., 2003; Tobias \& McNiel, 2008). The case presented was a long-standing one, as such, typical clinical manifestation of right heart failure exercise intolerance and classical radiographic findings of globoid cardiac silhouette were manifested. Analysis of the effusate did not incriminate frank blood as would have been seen in traumatic aetiology, anticoagulant toxicosis or cardiac neoplasia, (Petrus \& Henik, 1999; Prosek et al., 2003), or exudates as would have been seen in infections pericardial effusion, (Carpenter et al., 2001).

Radiography and echocardiography have been used to confirm pericardial effusion, and our findings agree with findings of other authors that reported on chronic pericardial effusion in dogs, (Tobias, 2005).

Emergency management of patients with confirmed pericardial effusion is pericardiocentesis, (Petrus \&

\section{References}

Aroshon LR \& Carpenter JL (1999). Surgical treatment of idiopathic pericardial effusion in the dog: 25 cases (1978-1993). Journal of American Hospital Association, 35:521-525.

Carpenter DH Jr., Mackin AJ \& Gellasch KL (2001). Cardiac tamponade secondary to Aspergillus niger induced constructive pericarditis. Journal of American Veterinary Medical Association, 218:1890-1892.

Heinritz CK, Gilson SD \& Soderstorm MJ (2005). Subtotal pericardiotomy and epicardial excision for treatment of coccidiodomycosis induced effusive constructive pericarditis in dogs: 17 cases (1999-2003). Journal of American Veterinary Medical Association, 227:435-440.

Petrus DJ \& Henik RA (1999). Pericardial effusion and cardiac tamponade secondary to brodificoum toxicosis in a dog. Journal of American Veterinary Medical Association, 215:647-648.

Prosek R, Sisson DD \& Oyama MA (2003). Pericardial effusion with a clot in the pericardial space likely caused by left atrial rupture secondary to mitral regurgitation. Journal of American Veterinary Medical Association, 222:441-442.
Henik, 1999; Tobias, 2005). This palliative management yielded good results in patients presented with idiopathic pericardial effusion in dogs, (Aronshon \& Carpenter, 1999). Where there are associated aetiologies, adjunct procedures were carried out that included, pericariotomy, sub-total pericardiectomy, tumor debulking \& chemotherapy, (Walsh et al., 1999; Carpenter et al., 2001 Heinritz et al., 2005; Tobias, 2005; Tobias \& McNiel, 2008).

The case managed responded to the palliative management of pericardiocentesis and did not need other adjunct procedures. The ultrasound guided pericardiocentesis afforded a visual, minimal invasive technique that prevented accidental contact with epicardium with the resultant cardiac arrythmia. An alternative technique is fluoroscopy, but this has massive exposure of the operator and patient to primary X-ray beam; there is also poor manipulation of the over-the needle catheter because of the use of lead gloves. Ultrasound guided pericardiocentesis is therefore advocated when pericardiocentesis is to be performed as an emergency or palliative management of pericardial effusion in dogs

Schelling CG, Saunders HM \& Workman JA (1993). Veterinary ultrasound atlas: Abdomen and thorax case studies of the dog and cats Corometrics Medical Systems Inc. Pp 123.

Sisson D \& Thomas WP (1999). Pericardial disease and cardiac tumors. In: Textbook of canine and feline cardiology, $2^{\text {nd }}$ edition (PR Fox, D Sisson \& NS Moise editors). WB Saunders. Philadephia pp. 679-701.

Tobias AH (2005). Pericardial Disorders. In: Textbook of veterianry internal medicine. $6^{\text {th }}$ Edition, (SJ Ettingers \& ED Feldman, editors) WB Saunders. St. Louis, Mo. Pp 1104-1118.

Tobias AH \& McNiel EA (2008). Pericardial disorders and cardiac tumors. In: Manual of Canine and Feline Cardiology. $4^{\text {th }}$ Edition, (T Smith (Jr), Oyama \& Leeper, editors) Saunders Elsevier, St. Loius Mo. 63146. Pp 200-214.

Walsh JP, Remediuos AM \& Ferguson JF (1999). Thoracoscopic versus open paratila pericardectomy in dogs: Comparison of post operative pain and morbidity. Veterinary Surgery, 28: 472-475. 